\title{
Based on Neural Network Information Fusion System Architecture Evolution Research in the Theory of Evolution
}

\author{
WU Rong-chun ${ }^{1}$, Zhang Feng-li and Nie Ben-jun \\ ${ }^{1}$ School of Computer Science and Engineering, University of Electronic Science \\ and Technology of China Chengdu 611731; Police Academy, China Chengdu \\ ${ }^{2}$ School of Computer Science \& Engineering, University of Electronic Science and \\ Technology of China Chengdu \\ ${ }^{3}$ Police Academy, China Chengdu \\ 610213,wjwrc@163.com,611731fzhang@uestc.edu.cn,179415437@9g.com
}

\begin{abstract}
Under the environment of the human nervous systen 2 symuti-sensor information fusion architecture for the most flexible. Papers through on people cranial nerve Yuan of evolution course and information fusion system Eusion unit of evolution process of compared research, proposed has a distribued information fusion system of evolution system structure, the system structure is information fusionsystem structure evolution of a senior form, it can according to network system different of mission, and external environment and network system of State automutically formed currently by knows of most effective of information fusion system structure, more effective to reached fusion purposes.
\end{abstract}

Keywords: Information Fusion, Network system, Architecture, Evolution, Internal Model

\section{Introduction}

Most studied at present, the most mature information fusion system architecture consists of a hode is a powerful operation and processing capacity to deal with all the data at the center of the type information fusion system structure. Theoretically, the original data of the node for each monitoring node by the center for fusion processing method is optimal, but modern information fusion system processing range is more and more big, more and more sensors and sources of information, more decentralized, although communication system development soon, the center node processing power, also can't for all da@ real-time transmission and processing in time, so the architecture is clearly unable to meet the increasing number of sensors and the information of communication bandwidth and information fusion system processing power put forward strict requirements.

Biological evolution has made many senior animals for a long time, especially our own, already has such flexible information fusion architecture, although we for their specific way of fabric, processing method and work mechanism is not clear. Because of the human central nervous system can according to different needs at different levels to fusion of information from facial features, real-time fusion results are obtained, and promptly make the optimal decision, command the corresponding parts of the body (actuators) corresponding measures; Experts also can get limited information, according to the rule of thumb for very complicated things to make the right judgment and take effective measures to achieve expected purpose. The basic starting point of this article is in the nervous system by drawing from neurobiology inheritance, development and evolution of research results, to guide information fusion system architecture design and application. 


\section{Nervous System Evolution}

\subsection{Nervous System Introduction to the Theory of Evolution}

Although we think discussed in multicellular animals nervous system seems to be reasonable, people, however, it is hard to imagine, in single-celled organisms also involves the signal mechanism of neuron function [1-2]. Such as protozoa paramecium could produce similar to the action potentials in neurons, although the action potential is due to the flow of calcium ions, rather like typical nerve action potential generation, is due to the presence of sodium ions flow. Similar to nerve action potential, action potential in the paramecia have some coordination function, regulate the direction of the cilium swings, so as to guide the paramecia move in different directions. Mutations of paramecium due to lack of ion channels, does not produce calcium ion action potential, keeping a cilia only resting state to the same direction, and thus when it encounters harmful chemical stimulus material can't swim back, and this mutation is called pawn. These facts indicate that neural signals from the evolution of the signal meghanism of single-celled animals modification process.

In multicellular animals lack of nervous system, canalso be found that the existence of early nerve signal system. Such as Water branches of cnidarians obelia, they have the coral samples columnar branch of the structure of the digestive system. Hydra has formed the first real nervous system. This is a very simple neural network system. Action potentials in spreading way, from one neuron along all đirections completely equal to another neuron. In this system, there is no any special neurons to control another, that is to say, any part of the neural network is not has a special function. These neurons are considered only in the animal bodyaction potential conduction pathways, like cnidarians obelia epithelial conduction systembug, it's just faster. Actually neurons, should be have nerve axons connected beam, arong a fairly@eccurate direction signals, rather than like the epithelial cell layer, there isno specific direction of conduction.

As animals' front-end ganglion xolume increases and the increase of complexity, ganglion fusion as an entity, this creates a primitive brain $[9,15]$. Despite the mammalian brain did not shomobyious neuron concentrated external trace, but in fact there are a lot of different group of neurons, called the nucleus and ganglion, their incoming or outgoing nerve fibers connection between each other [3]. In this way, the most basic of interconnected nerve chan formed the original structure of the brain.

By concentrated ganglion in the head what will surely lead to the head in the control of the body in an important position in the nervous system [12]. In the most basic of the nervous system, while information can flow from a ganglion to another ganglion, yet each segment ganglia just coordinate with a special body segment of behavior, it is not each other between the ganglion controls. However, with the emergence of head quarter and primitive brain, nervous system (hierarchical) form began to appear, head nerve for the body of the integration of sensory and motor information, and launched activities of the body, to produce all kinds of behavior. The trend appeared to be significant in vertebrates, in these animals in the central nervous system is further divided into the brain and spinal cord.

\subsection{Nervous System Evolution Trend Analysis}

As mentioned earlier, from single-celled organisms with similar functions to neurons in the nervous system mechanism of electrical signals, after a long evolutionary process, and gradually evolved into senior mammalian nervous system. Can find senior mammalian nervous system evolution trend of basic fabric for the following four aspects:

(1) From the evolution to bilateral symmetrical radial symmetry;

(2) The evolution of the individual neurons from the more general to the specific function;

(3) The head of focus; 
(4) The classification of fabric.

This evolutionary process can be divided into four main stages:

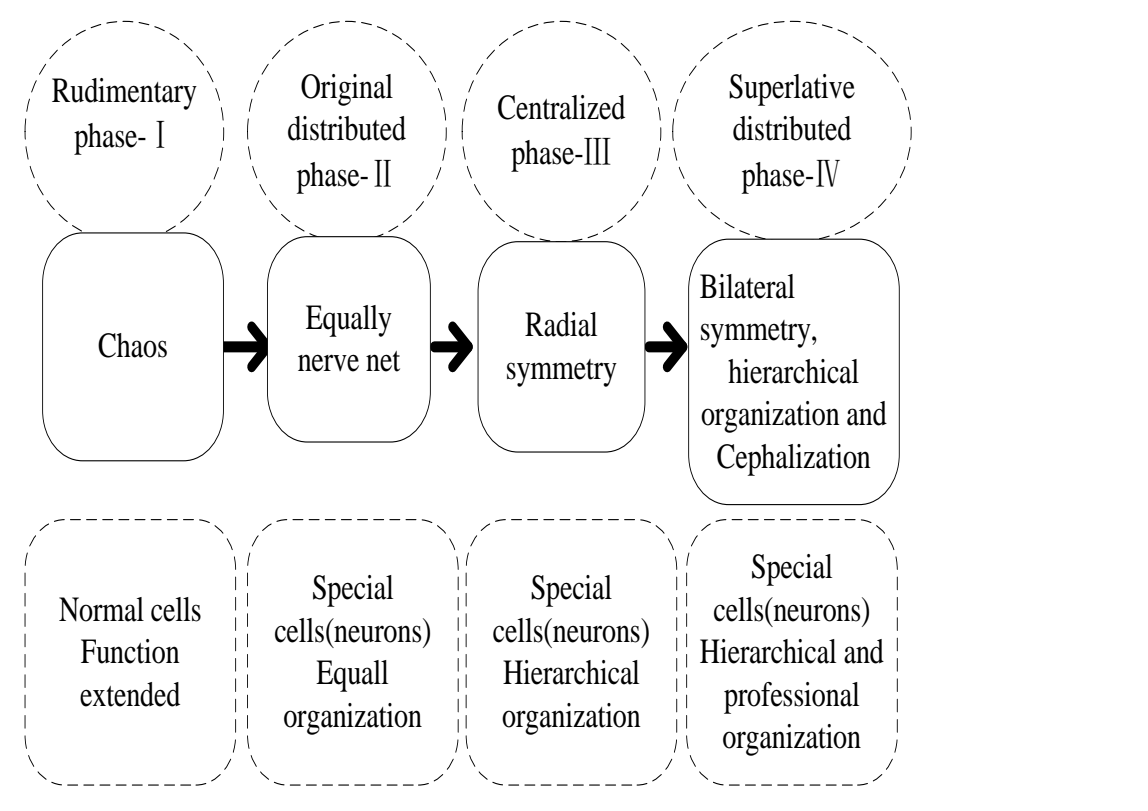

Figure 1. Four Evolution Phases of Nerve Net and Neuron Organization

In the first stage of not fully differentiation, the sense of environment and organism control function is made on some normal cells in the functional development of expansion, control structure berongs to a state of chaos; As a professional feeling and control functions of nerve cells (neurons), formed a parity of the structure of the original distributed neural network, each neturon has no function difference, this is the second phase of evolution, With eentral type radial symmetry of the third stage is because in the special location, often the body in the middle of the further differentiation of neurons function, enhanced control processing functions and gradually form a hierarchical neural network; Center type level keural network architecture in accordance with the above four aspects the evolution rend of further differentiation development, finally gradually evolution to the fourth stage with advanced distributed system structure. Figure 1 shows the nervous system in the evolution of four phases, as well as the corresponding neuron function and organizational structure.

\section{Fusion Unit}

Nervous system evolution and the development process of information fusion systems have many similar characteristics [8], therefore, draw lessons from the evolution of the nervous system helps information integration system design and development. First of all, unit structure and the characteristics of information integration system and the characteristics of the neurons in different stages of evolution in comparison may be necessary. As discussed below, the function, structure and information fusion node almost as well as the role of neurons in the nervous system. It is for this reason, in order to compare convenience, also called information fusion unit.

As to be able to accept and response signal and stimulate the sensor, and the emergence of signal processor, appeared such as analog/digital signal processing, state estimation, control theory and artificial intelligence, help a single sensor performance technologies such as these technologies were also applied to the more advanced stage of information fusion. So you can see information fusion technology is derived from sensors and a processor structure, appeared before the first information fusion system of chaos phase. 
The earliest information fusion system is mainly for military application. When we will be more sensors, such as multiple radar or sonar, joined together to guide each other, would seem to form a fusion system at the earliest. At this stage, the sensors usually belong to the same type, and no sensor has priority, as first II stage of neuron in the course of evolution. During this period, such as communication function becomes a processor has an important function, at the same time in order to combining sensor has a time delay of data also need to establish a dynamic data storage and management mechanism (database management system). Therefore Fusion unit contains three parts: at this stage the Fusion processor, Exchange and database management system (DBS), it is the structure of FED.

The function of switch is in the information fusion between system and environment as the sensor and transmitter. Distributed data management system was originally used to store and retrieve data and information about the sensor parameters and text based fysion results and algorithms such as template sample set components [13]. With the development of information fusion technology and database technology, distributed data management system plays a more expanded role Finally, the distributed data management system developed into a data mart or warehouse the eontrol methods of may for hierarchical or as a hierarchical structure.

Along with the new sensors and advanced processing teehnology and processing the development of hardware technology, distributed network real-time information fusion is becoming more and more possible [5-6]. Communication and resource allocation become a highlight the important issues. With high performance, Yow price of microprocessors and embedded computer, Intelligent Interlace is separated from fusion processor and become an independent part of the merging unit. So, form included in information fusion center, switch, database module and the intelligent interface of a fusion unit.

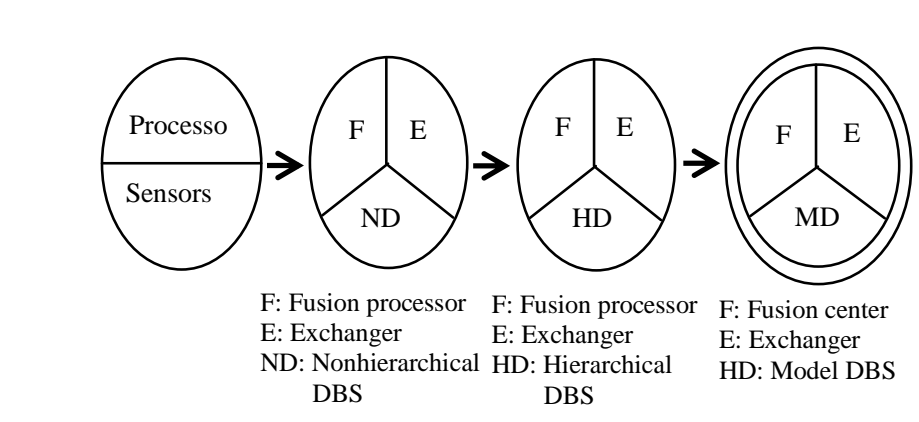

\section{Figure 2. The Four Kinds of Styles of Data Fusion Units}

Figure 2 for the evolution of fusion unit. According to the structure of database management system the FED structure is divided into two stages. Before one phase with the hierarchical structure of the database management system, the corresponding neurons II place for evolution stage, after one phase has hierarchical database structure, and the evolution of the neuron phase III corresponding. Here the database is not limited to the traditional database management system, can also be distributed database system, data marts or data warehouses, etc., so fusion unit itself may be a more complicated equipment or network, can be registered it is information integration system of hardware and software resources, collaboration and initiated by information fusion network or other fusion nodes information fusion task of units.

\section{Information Fusion System Architecture of Evolution}

The existence of a variety of data sources and fusion unit for information fusion architecture provides a variety of options, that is to say, you can through different 
ways of organization unit between the sensor fusion, fusion unit of data transmission mode and connectivity. There are four architectures: fully distributed, center, feedback rating and no feedback type [12]. From the previous analysis we discover not hard in the course of evolution of the fusion cells and neurons, they have the astonishing similarities. Thus we can build a new information fusion system architecture based on evolutionary point of view. As a natural result of merging unit evolution, the evolution of the information fusion system architecture form for four stages.

When the sensor information in time or space to get the rough processing, forming the first stage, as shown in Figure $3(\mathrm{a}, \mathrm{b})$. Along with the development of the processor performance and sensor resolution, gradually formed the local database, so get a processor contains integration, switches and the integration of database (FED) structure unit, as shown in Figure 3 (b). Fusion process, on the basis of chaos its performance mainly depends on the integration of the performance of the sensor.

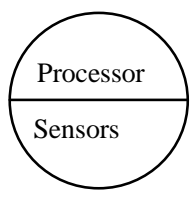

(a) prototype

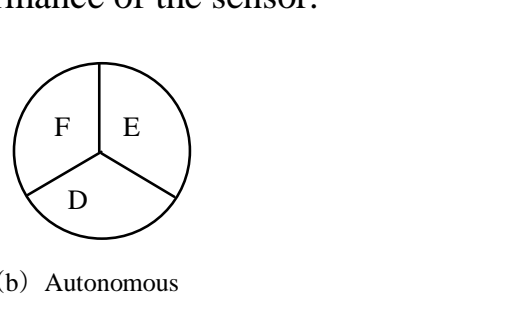

\section{Figure 3. The Evolutionary Architectures of Data Fusion System}

Along with the development of computer, communication technology and information fusion algorithm and fusion some units, such as radar are linked to each other, thus forming the first real information fusion system, the information fusion system architecture of the second phase, as shown in Figure 4. This is real distributed information fusion system architecture, integration between units without hierarchy, each node is only bound by the connectivity between the communications, and can be synchronous communication. This stage database belongs to a kind of distributed hierarchical structures.

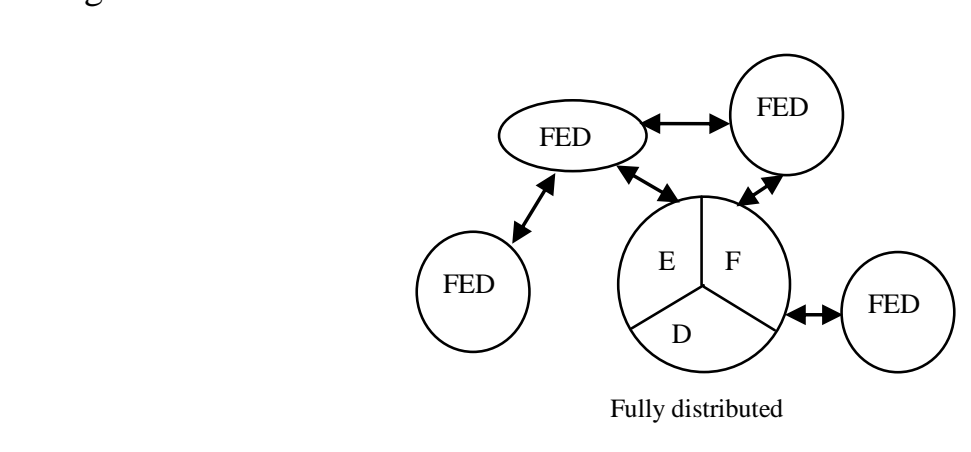

Figure 4. The Evolutionary Architectures of Data Fusion System

Third stage of evolutionary architecture as the center type architecture is a high performance computer system and the effective information fusion algorithm of complex product, as shown in figure 5. Center type architecture contains a unit has a unique position at the center of the fusion, at this stage consists of a single fusion results database, data from different sensors here after calibration and correlation and used to update the track and target state estimation. This one phase of the database has developed into a kind of distributed hierarchical structure, in fusion center unit maintains a global target track, but in all other fusion unit maintains only temporary track data. 


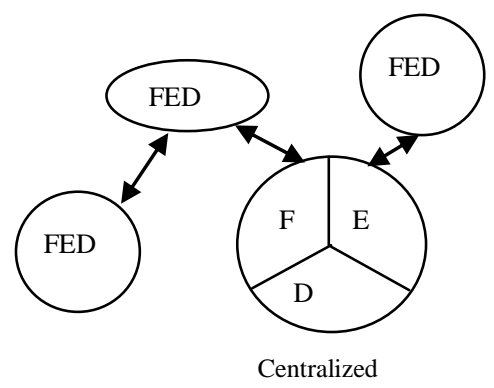

\section{Figure 5. The Evolutionary Architectures of Data Fusion System}

Information fusion system evolution system is one of the last stages for the hierarchical architecture, as shown in Figure $6(a, b)$. The system structure of information fitsion nodes are arranged into a hierarchical structure, higher level node processing tesults from a lower level node and could provide some feedback. In many applications it is a very natural architecture, for example, a node processing radar data, otherwise a node processing infrared sensor data, and a node is used to track data of radar and infrared sensor node merging into a final track as a result. If the data is transmitted at a rate below the sensor observations to a higher level of fusion node, can be very good save communication resources. If have feedback system of hierarchical structures will feedback the result to each node, is equivalent to a periodicbroadcast system.

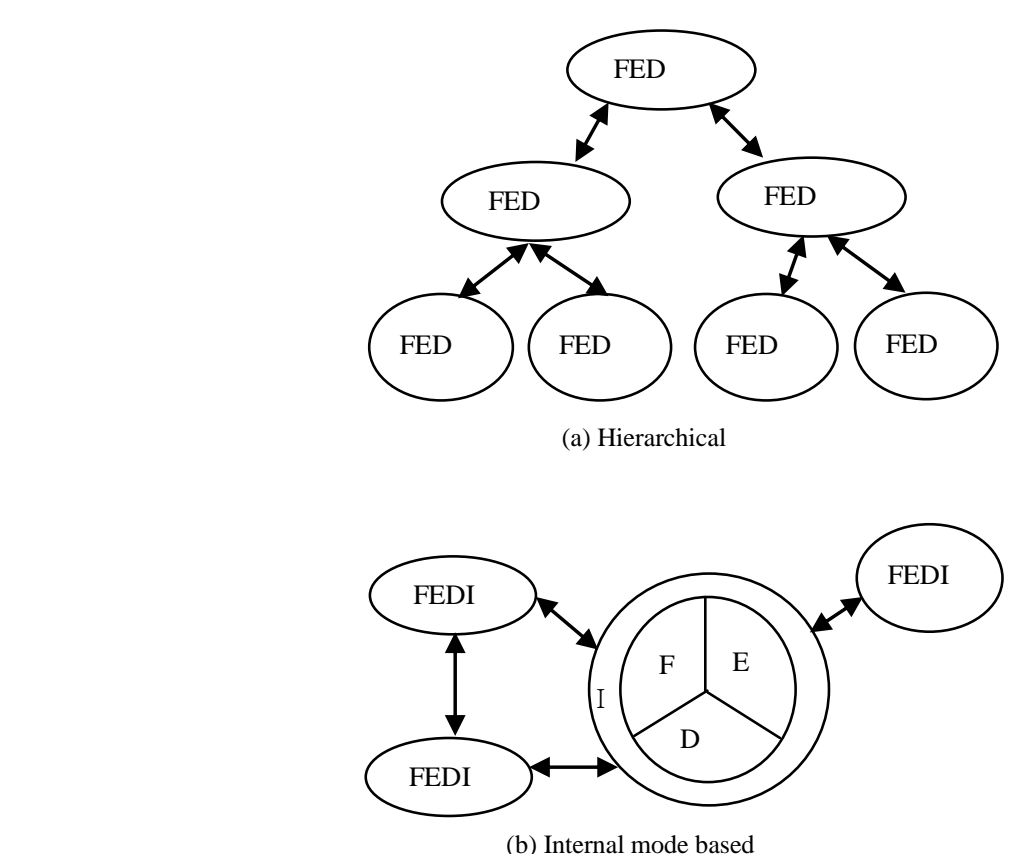

Figure 6. The Evolutionary Architectures of Data Fusion System

Fusion stage 4 (FEDI) is used to constitute another form of hierarchical architecture, called hierarchical evolutionary system based on internal model structure, as shown in Figure 6 (b). In this evolutionary system structure, formation of grade is determined by internal model of a kind of dynamic structure, the internal model is integrated with expert knowledge, history and successful experience, command decision-making command and environmental monitoring results, etc. and after formation of the uncertain information. 


\section{Internal Model and Driver of Information Fusion System}

\subsection{Internal Model of Information Fusion System}

Hierarchical information fusion system based on internal model structure form more like in the lower stage of fully distributed architecture, rather than at the center of higher phase type and hierarchical architecture phase. In FEDI structure, the FED's core more like neurons in the nucleus, its function includes: real-time data processing, to maintain normal function, data exchange and update, the historical data of learning and memory, as well as the internal model of storage, etc. And intelligent interface is more like neuron synapses; its main tasks include the network processing functions, signal transition and transfer, information selection, resource allocation and network instruction execution/and so on. Signals transition is to complete the outside world and the FED's core fincluding control signal and information, etc.) the process of transition, signa transfer is an information node to receive, store, and send the transferinformation.

Based on FEDI network, can provide very flexible mechanism for information fusion system is used to imitate the human nervous system in multisensory environment information processing of the most successful examples. Humah nervous system in general fusion from five sense organs of five types of signals (sight, hearing, smell, taste, touch) and near real time optimal decision-máking is given. According to different functions, the integration can occur through different combinations in different level; therefore the human nervous systemfdoes represent a very flexible architecture of information fusion.

Scan of the internal model for sensory organs, gaze changes, attention and touch, such as movement, has the stability of the timescale. Sensory input will eventually update and refine internal model. An organism to external stimuli response not only because of the instant input, more major is based on internal model has been established. The information processing mechanism is particularly suitable for based on internal model is used to improve the information fusion system with FEDI fusion unit performance. Simulation in order to achieve such an effect, the first question is on the information fusion system to establish a dynamic model of internal memory is used to represent and external observation and event information from the surrounding environment. This internal model should include not only traditional database function, still should have learn from database to store data and mining the useful information ability, information extraction and matching memory response to the external phenomenon. And, of course, under the environment of distributed multi-sensor information fusion, the model should also is a distributed structure model.

Operating system software is the basis of this new type of information fusion system structare. Considering the recycling system already has hardware and software resources, different development background, environment, and the way of managers, work units merge core the FED will be permitted to own a variety of different sources of operating system. This design allows developers in existing systems development with new services and missions applications, application and ability to quickly respond to the changing environment. It can facilitate the implementation of the existing system upgrades and will integrate new fusion unit.

\subsection{Information Fusion System Internal Model of the Drive}

It's believed to nerve tissue memories are stored in the synapse between neurons, and form a connection matrix [10]. The time stability of the connection between neurons are not the same. Presumably, long-term memory is based on the anatomy of formation and stable structure. A similar mechanism can be applied to information fusion system based on internal model. Internal model of long-term memory isstored in distributed to the local 
database, only the selected internal model, after being transferred to network in intelligent interface template as a dynamic operation, by the fusion of the template should organize units to work together.

Just like the neurons of the nervous system, merging unit can present any dynamic or inactive state, but the activity of these units is interdependent. Fusion unit results received from the switch will be continuously evaluation by local data mart. Once the data mart find interested events, for example, found that some kind of threat, it will choose a suitable internal model to deal with these threats, and then notify the intelligent interface on the information fusion network load and run the internal model. Fusion unit may be related to the operation of the internal model of activation, and the corresponding results feedback to launch event fusion unit or another designated unit for further processing. Obviously, this is used for processing unit can become a information fusion center, at the same time also suggests that information fusion system based on internal model structure is a hierarchical system. Due to the interface section separated from information fusion core, so every fusion unit may be in three states: active mode, cooperation mode and the inactive state. Figure 7 is a fusion of unit three states in an application mstance of the fusion system with grid connection.

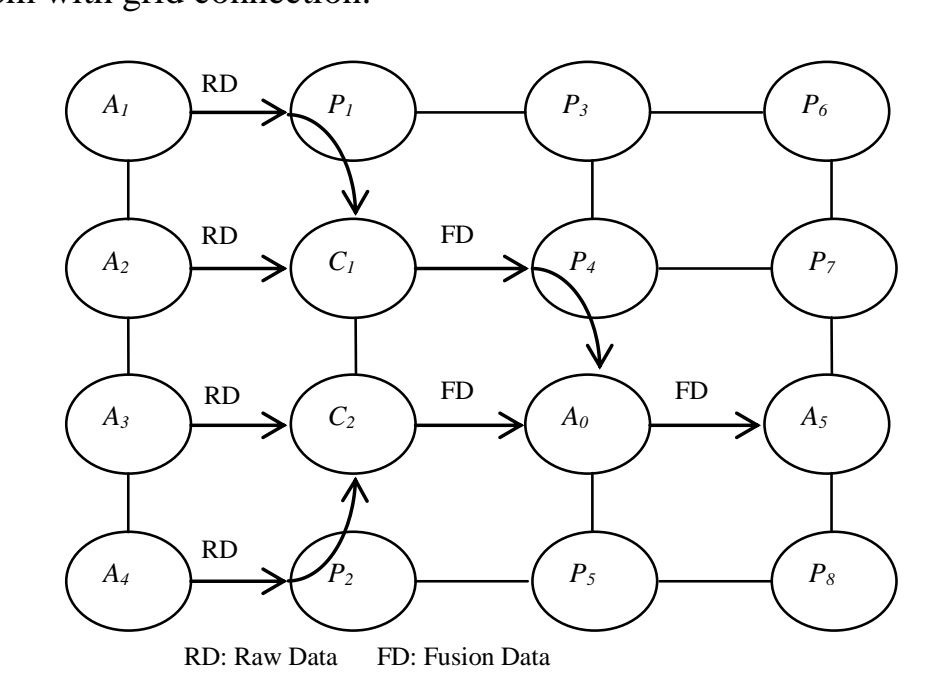

Figure 7. Three Stages of Data Fusion Units in an Internal Model based Data Fusion System

In Figure 7 the fusion events are launched by A0. A1, A2, A3, A4 and A5 these five awakened become activated state fusion unit. $\mathrm{C} 1$ and $\mathrm{C} 2$ are awakened to become state of cooperation, other cells in the inactive state. $\mathrm{C} 1$ and $\mathrm{C} 2$ in collaboration with $\mathrm{A} 0$ processing raw data from $\mathrm{A} 1$ to $\mathrm{A} 4$, and their fusion results are sent to the $\mathrm{A} 5$ for further processing. However, despite the P1, P2 and P4 units in inactive state, but because they use their own intelligence interface involved in transmitting data, therefore, also they can be classified as cooperation unit.

Nervous system constantly by stimuli from the environment changes, the stimulus will be mandatory to raise the input and output elements form a certain activity, through restrictions likely to generate the output elements, thus affecting output [26]. Because there are a plenty of contact with each other between the constituent elements, so after stimulation the entire system can change its internal state. If there is certain inherent learning rule, these rules in the process of repeated activities form elements can modify connection strength between, so network can memory external stimuli. If the external shape is not random, but has the certain regularity, network will form the storage form of arrays, each array will have obvious difference. Similar to this process is suitable for internal information integration system is used to produce and memory model [14]. In 
addition, sometimes may be managers or expert system to evaluate the result of the operation and will feedback the result to the fusion system to form internal model.

Based on Information fusion system internal model can not only build on existing based on multiple information fusion system, and the dynamic hierarchical fusion architecture can construct a variety of data sources and unit information fusion architecture, it will also be able to decide when to fusion of the observed event, how to get the most useful information (sensor resource management), and how to integrate information (resource allocation).

\section{Examples of Application}

This above information fusion system architecture evolution research, its main purpose is to provide a generic and efficient integration and design platform. In this a section in the we first established a digital of battlefield environment, simulation fleet (Red Army) in the monitoring environment Xia on enemy machipe (Blue Army) and by launches of missile and torpedo of fulf process, formed a virtual interference monitoring environment, as evolution type information fusion system structure in complex environment Xia of application instance, take this analysis internal model of run and processing mechanism, and various stralegy thought in the environment Xia of using method.

\subsection{Digital Establishment of Naval Combat Environment}

A red side and a blue side troop strength and deployment.

Red party information will be included in the converged environment the following systems:

A flagship has on its search radar, muttiple target tracking radar, infrared video tracking system, communication and identily identification system;

Number of frigates, It has the target tracking radar, fire fighting system, communications and didentity on discernible system, etc.

Blue party information wifl be included in the converged environment the following system

The blues are equippect with a bomber, the blues will to lob missiles in red and precision guided torpedo each one.

Both sides have their own satellite-based network consisting of support, complete communications and positioning and precision guidance capabilities.

Without loss of generality, set Red's flagship is located at the coordinate origin, fire hit back at East flagship 5-kilometer of the frigate completed and for the $\mathrm{X}$ axis to the East, North to the $\mathrm{Y}$ axis, $\mathrm{Z}$ axis points to the zenith.

Other parameters are as follows:

Blae bombers from the Northeast by 8-kilometer height to the red flag flew with $350 \mathrm{~m} / \mathrm{s}$ speed, after the firing of a missile and torpedo, 1-kilometer radius semicircular track climb to 10,000 feet along the invasion have fled.

Red search radar $(30000,30000,8000)$ (units of meters, when without confusion, without indicating the units are meters) and found an unidentified flying object (that is, the blue bombers), information fusion event that is as time zero. Blue Bombers fired one air-to-ship missiles after 20 seconds, 45 seconds later, throwing a precision-guided torpedo missile in his throwing motion in 4 seconds after ignition, $180 \mathrm{~m} / \mathrm{S} 2$ horizontal acceleration speed for 2 seconds turn off the engine, then a throwing motion, after the frigate destroyed by red. Fish Lei Zapping throwing movement, also were destroyed by the Red Guard. Level bombers to escape were also destroyed in the process.

Establishment of target observation model: consideration of hybrid system of linear equation of State and measurement description. 


$$
\begin{aligned}
& x(k+1)=F(k) x(k)+v(k) \quad k=0,1,2, \ldots \ldots \\
& z(k)=H(k) x(k)+w(k) \quad k=0,1,2, \ldots \ldots
\end{aligned}
$$

Among them, the ${ }^{x(k)}$ represents the State vector ${ }^{k}$ goals, ${ }^{z(k)}$ means ${ }^{k}$ vector of observations, $F(k)$ means that the State transition matrix, ${ }^{H}(k)$ means that the measurement matrix, ${ }^{v(k)}$ and ${ }^{w(k)}$ is a zero mean independent white Gaussian noise.

Figure 8 no noise in the Cartesian coordinate system, respectively original data and adding noise objectives under the three-dimensional State vector. Figure 9 is a target bearing, height and distance of measurement space simulation data. This environment can be used for simulation and test integrated in the effectiveness of information fusion algorithms evolutionary architecture, selection and simulation of fusion algorithm is beyond the scope of this article, the following analysis of naval confrontations both during working process of information fusion system,

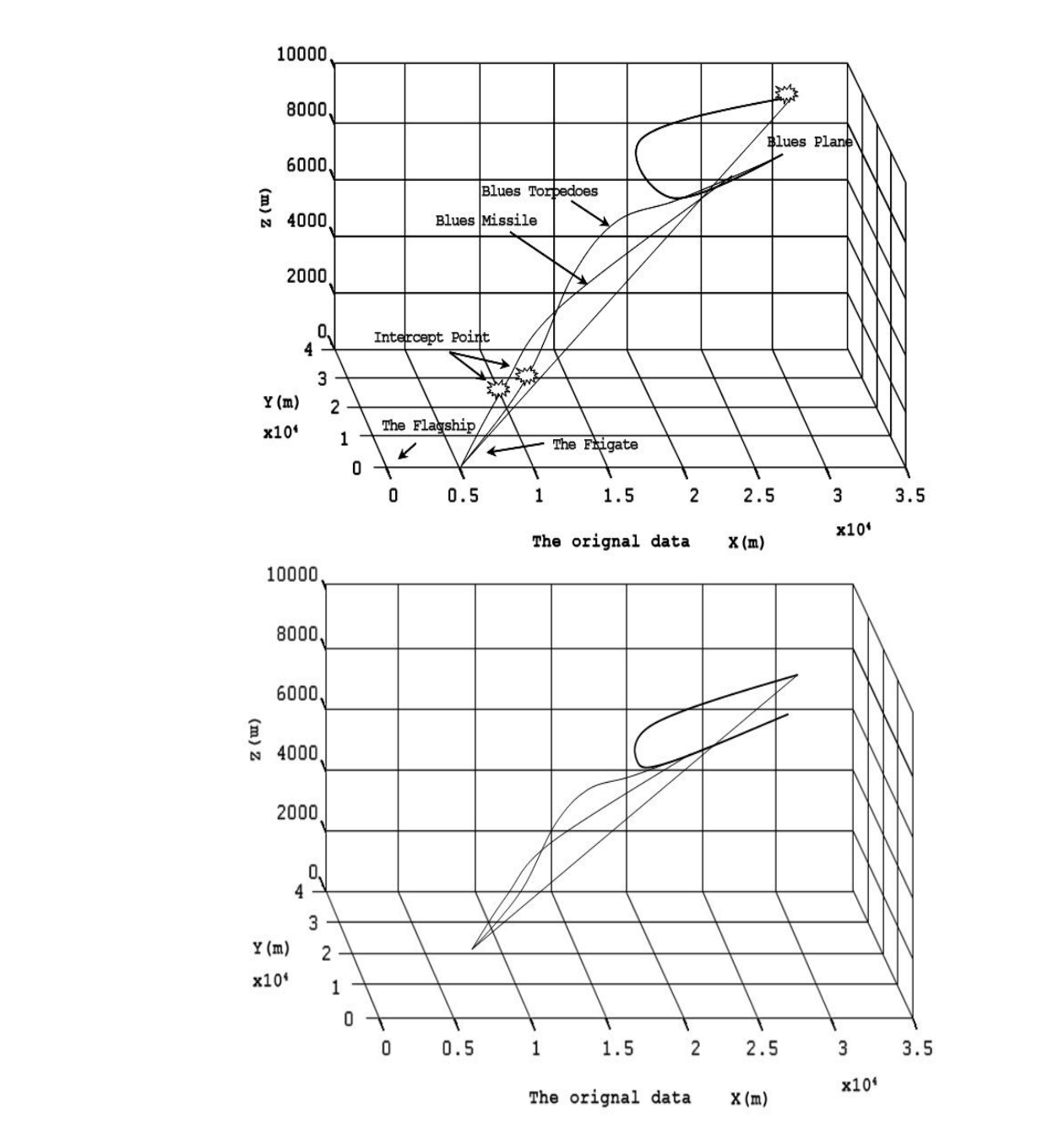

Figure 8. The Original Data and State Space Data in Cartesian Coordinate 

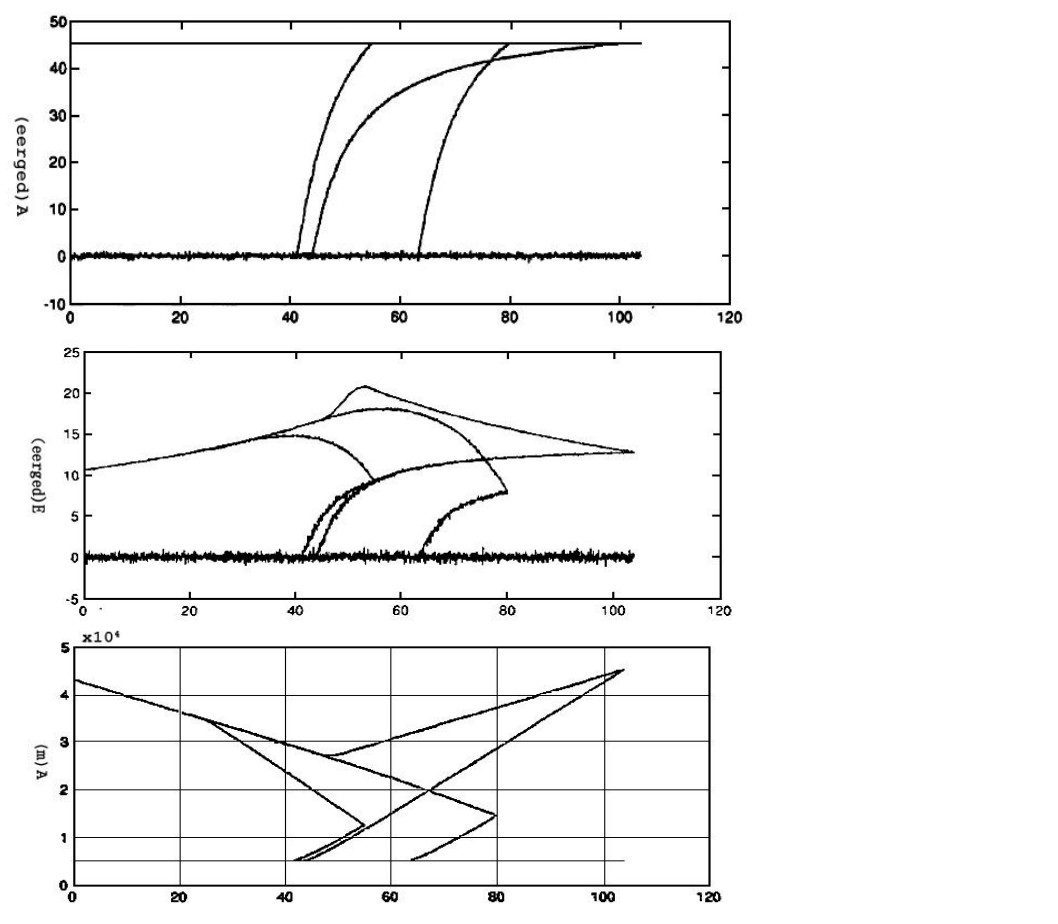

Figure 9. Measurement Space A,E and R Data Respect to $t$

\subsection{Naval Confrontation in the Course of Intêrnal Models}

Red flag on the determination of multiple targets in multiple target tracking radar bearing and distance, infrared Miss distance in TV tracking system can extract multiple targets, so as to locate targets and high and low angle, assuming that their sample rate are $20 \mathrm{C} / \mathrm{S}$, communication and identity identification system to receive all red target and fire. Convoy by radar-guided system can lock the blue target on the ship and to intercept missile can be precision-guided. Both space-based networks will be on the individual communications, positioning and precision targeting support.

Here, red side information integration of network systems hardware and software resources including its flagship command center, the frigate command centers, radars and infrared tracking system and other sensors, communications networks, identity recognition system, fire support and command system, data warehouse, information and information processing sectors, as well as officers and men at all levels, and so on. Network information fusion for integrated decision-making proces is saved in the data warehouse knowledge and command of the command (Destination), to observe and gather information to make the right decisions, and take corresponding countermeasures, or should I say in the selection of the appropriate model from the data warehouse and keep it running correctly on the network.

Normally, red information fusion network is comprehensive monitoring status. Popular Party flagship Shang of warning radar system found air appeared a new target, on will automatically adjustment network run of internal model, this situation for system by known situation, processing method for requests target identity identification system and target track determines module collaboration, such as, once according to fuzzy Hough transform, determines for new target track, and identity identification system cannot verified its identity, is immediately started on unknown identity flight property warning processing model.

Unidentified flying objects-warning models will use the following resources: start passive infrared or radar system to achieve the objectives of close surveillance, 
tracking and positioning, target identity recognition technology on a new target for identity verification, notify the fire system personnel in place, just in case, as well as through direct network to report the situation and so on.

Selection of internal model will also use some a priori knowledge of the data warehouse. In on identity unknown of flight property monitoring process in the found near and appeared new target, began started more target captured and track module, fuzzy logic agent according to store in data warehouse in the of prior knowledge and more target captured and track module of fusion results judgment is may is enemy launches of attack missile, target, new target in is not ignition simian track should for flat throwing movement, if its track head-on to we flew, is judgment for aircraft to we launches of bomb, and torpedo or missile, should immediately on its for intercept; Even if it is not for us, but found the ignition, because missiles can be Omni-directional attack, is also identified as an enemy target, should inform the network transferred operations to intercept enemyrtarget model of information fusion.

Intercept enemy target of combat model run process in the, red $\hat{p}$ arty of all target are can through identity identification system obtained its physical and dynamics parameter, red party communications resources of priority level by fuzzy logic agent according to task changes dynamic decided finepower automatically command decision-making process following: in confirmed aircraft identity and found it too we launched attack Hour, immediately notification frigate firepower command system run intercept target module, which enemy target priority level decided by fuzzy logic agent. 4 seconds later foind its ignition speed up coming to us, confirm its information fusion system for aircraft launch missile, immediately ordered the convoy to intercept. Because of the missile threat, high priority, the first interceptor missiles and attack aircraft. Interceptor missile in the course of, flagship enemy aircraft fired another fliey from data warehousing knowledge information fusion system, missile or bomb ordered the convoy to intercept and attack aircraft.

In Table 1 and Table 2 lists the change situation of the internal model in the process of operation.

\section{Table 1. The Change Procedure of Internal Model of the Red in the Counteract}

\begin{tabular}{|c|c|c|c|}
\hline Triggering event & Fusion (Network) & Internal models & Battle fusion (network) \\
\hline Command & Commander & Pre-ring model & $\begin{array}{l}\text { Space-based networks on board, early warning radar } \\
\text { and observation posts }\end{array}$ \\
\hline Find & Surveillance radar & Identity network & $\begin{array}{l}\text { Space-based networks, multiple target tracking and } \\
\text { measuring networks on each ship, identification } \\
\text { network, fire control systems, and command system }\end{array}$ \\
\hline & Identity network & $\begin{array}{l}\text { Operational } \\
\text { model }\end{array}$ & $\begin{array}{l}\text { Space-based networks, multiple target tracking and } \\
\text { measuring networks on each ship, identification } \\
\text { network, fire control systems, chain of command, } \\
\text { prioritize fuzzy proxy }\end{array}$ \\
\hline $\begin{array}{l}\text { Aircraft launch } \\
\text { missile }\end{array}$ & $\begin{array}{l}\text { Multiple target } \\
\text { tracking and } \\
\text { measuring networks }\end{array}$ & Fire model & $\begin{array}{l}\text { Space-based networks, multiple target tracking and } \\
\text { measuring networks on each ship, identification } \\
\text { network, fire control systems, chain of command, } \\
\text { prioritize fuzzy proxy }\end{array}$ \\
\hline $\begin{array}{l}\text { Aircraft ejected } \\
\text { the torpedo }\end{array}$ & $\begin{array}{l}\text { Multiple target } \\
\text { tracking and } \\
\text { measuring networks }\end{array}$ & Fire model & $\begin{array}{l}\text { Space-based networks, multiple target tracking and } \\
\text { measuring networks on each ship, identification } \\
\text { network, fire control systems, chain of command, } \\
\text { prioritize fuzzy proxy }\end{array}$ \\
\hline $\begin{array}{l}\text { Plane to turn } \\
\text { around }\end{array}$ & $\begin{array}{l}\text { Multiple target } \\
\text { tracking and } \\
\text { measuring networks }\end{array}$ & Fire model & $\begin{array}{l}\text { Space-based networks, multiple target tracking and } \\
\text { measuring networks on each ship, identification } \\
\text { network, fire control systems, and command system }\end{array}$ \\
\hline The end of the war & $\begin{array}{l}\text { Multiple target } \\
\text { tracking and } \\
\text { measuring networks }\end{array}$ & $\begin{array}{l}\text { Early warning } \\
\text { model }\end{array}$ & $\begin{array}{l}\text { Space-based networks on board, early warning radar } \\
\text { and observation posts }\end{array}$ \\
\hline
\end{tabular}




\section{Table 2. The Change Procedure of Internal Model of the Blue in the} Counteract

\begin{tabular}{l|l|l|l}
\hline Triggering event & Fusion (Network) & Internal models & Battle fusion (network) \\
\hline Command & Commander & Target acquisition model & Pilot system, based on the network, search radar \\
\hline $\begin{array}{l}\text { Found the enemy } \\
\text { ship group }\end{array}$ & Search radar & Attack model & $\begin{array}{l}\text { Pilot systems, space-based networks, which control } \\
\text { radar, missile launching system, identification, } \\
\text { electronic warfare networks, the chain of command }\end{array}$ \\
\hline $\begin{array}{l}\text { Enemy radar } \\
\text { locks }\end{array}$ & $\begin{array}{l}\text { Electronic } \\
\text { warfare network }\end{array}$ & $\begin{array}{l}\text { Accelerated attacks } \\
\text { preparatory retreat } \\
\text { model }\end{array}$ & $\begin{array}{l}\text { Pilot systems, space-based networks, which control } \\
\text { radar, missile launching system, identification, } \\
\text { electronic warfare networks, the chain of command }\end{array}$ \\
\hline Attack is over & $\begin{array}{l}\text { The fire control } \\
\text { system }\end{array}$ & $\begin{array}{l}\text { Pilot systems, space-based networks, electronic } \\
\text { warfare network }\end{array}$ \\
\hline
\end{tabular}

Confirm the new status to complete the interception of missiles and torpedoes, and total time less than a minute, if the commanders tell the information, and make decisions, and how difficult it can be imagined, in the trenches of the enemy may achieve a close range attacks, and therefore permitted $\langle$ by, the corresponding time will be shortened. Information fusion system architecture will be the evolution of the decision-making process becomes automatic model selection and operating process, rapid decision-making to achieve this sequence prøvides, a new approach.

\subsection{Model of Evaluation and Internal Refining.}

Situational evaluation capacity and command and control capabilities, operational effectiveness is the key to winning future wars, Cncluding space-based, air-based, sea-based and ground reconnaissance, surveillance, early warning, navigation, and the like are important means to ensure effective use of these abilities. Combat command and information edhnology management personnel in pre-war, and battlefield and post-war according to posture perception system results on by using of or may using of internal model for effect assessment, data warehouse model management module is according to dssessment results achieved on internal model increased, and modified or scouring, to this achieved on data warehouse in the by Save internat nodel of upgrade, achieved has model of evolution, will greatly improve network of combat and strain capacity.

High resolution photography reconnaissance satellites, and next-generation days base infrared ballistia missile warning system, and air combat phase controlled array warning machine and air on to depth attack of monitored command aircraft, and unmanned reconnarssance and night depending on equipment wait has precise, and real-time, and clock combat capacity information gets means, will makes command decision-making personnel has global posture perception capacity, plus $3 \mathrm{G}$ and network technology of development, internal model resources of evolution and shared will in more big range within implementation, Play a role of information fusion system architecture evolution offers a wide range of space.

\section{Conclusions}

In the study of the nervous system evolution theory and analyzes its evolution trend, based on the unit and the internal model is proposed based on the fusion of two important concepts, and in this proposed on the basis of modern information fusion system architecture evolution. This architecture implements completely affirmative distributed architecture, from each according to his ability, to get, and imitate the human nervous system information fusion system.

Internal model of information fusion system is the network system in the process of adapting to the external environment through continuous learning, memory and refining of a sequence of decision-making model. Known as network running events 
or information fusion, is actually preferred an internal model to work on the Internet, the internal model determines the system structure which is formed by the system to work. Has intelligent interface fusion unit, it will be in charge of communications systems and dynamic resource management functions, which have the ability to run internal model, so that the core of fusion unit can be devoted to more important information such as mining, modeling and decision fusion task, at the same time also makes the evolution of the information fusion system architecture have intelligent networking ability, can according to its mission and environment of all kinds of best known architecture.

\section{Acknowledgements}

This work was supported by the National Science Foundation (Grant dio. 61133016), and the National High Technology Joint Research Program of China (863 Program, Grant No. 2011AA010706), thank for the help.

\section{References}

[1] M. E.Liggins II, C.-Y. Chong, I. Kadar and M. G. Alford, "Distríbuted Eusion Architectures and Algorithms for Target Tracking", PROCEEDINGS OF THE IEEE, vol. 85, no. 1, (2007) January, pp. 95-107.

[2] G. G. Mattherws, "Neurobiolog: molecules, ells, and systens", Blackwell science, Inc. Malden, Massachuseets, USA, (1998), pp. 1-36.

[3] V. L. Vvedensky, "Comon model of evolution for living cell and central nervous system", Bioelectrochemistry and Bioenergetics, Nol. 48, (1999), pp 343-347.

[4] N. Bisnik, A. Abouzeid and V. Isler, "Stochastic eventoapture using mobile sensors subject to a quality metric", In MobiCom, (2006).

[5] P. Brass, "Bounds on coverage and targetedetection capabilities for models of networks of mobile sensors", ACM Trans. Sen. Netw., vol. 3, no 2, (2007).

[6] E. Cayirci, W. Su and Y Sanl arasubramanian. "Wireless sensor networks: A survey. Comput. Netw.", vol. 38, no. 4, (2002) March, pp. 393-422.

[7] C. Jae-Hwan and T. Leandros, "Energy conserving routing in wireless ad-hoc networks", In Proceedings of IEEE INFOCOM (2000), pp. 22-31.

[8] R. Kumar, M. Wolenetz, B. Agarwalla, J. Shin, P. W. Hutto, A. Paul, and U. Ramachandran, "DFuse: Framework for distributed data fusion", In Proceedings of ACM SenSys2003, (2003).

[9] S. Purwar, I. N. Kar and A. N Jha, "On-line system identification of complex systems using Chebyshev neural networks [J]", Applied Soft Computing, (2007), vol. 7, no. 364.

[10] N. J. Higham, "Evaluating Pade approximants of the martrix logarithm [J]", SIAM J. Matrix Anal. APPl., vol. 25, (2003), pr 464-485.

[11] J. Chen, D. J. Dewitt, F. Tian and Y. Wang, "NiagaraCQ: A scalable continuous query system for internet/databases", In Proceedings of the ACM SIGMOD Conference on Management of Data, (2000).

[12] J. Liu, "Study on RBF Neural Network Improvement and its Application", Lanzhou University, Lanzhou (2008).

[13] C. Grille, L. Lppolito and V. Loia, "Agent-based architecture for designing hybrid control system”, Jifformation Science, vol. 176, (2006), pp. 1103-1130.

[14] G.F. Koob, "Dynamics of neuronal circuits in addiction: reward, antireward, and emotional memory", Pharmacopsychiatry 42 Suppl. 1S32-41, (2009).

[15] M. Jenkinson, P. Bannister, M. Brady and S. Smith, "Improved optimization for the robust and accurate linear registration and motion correction of brain images", Neuroimage, vol. 17, (2002), pp. 825-841. 


\section{Authors}

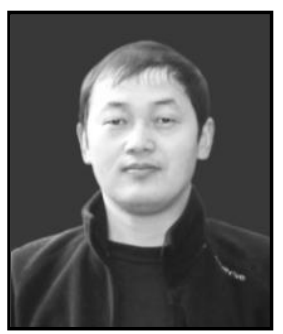

Wu Rong-chun, male, born on 6th, December, 1976, Han nationality, doctoral candidates, works at Police Academy, lecturer, main research directions: Information fusion, command automation. E-mail:wjwrc@163.com

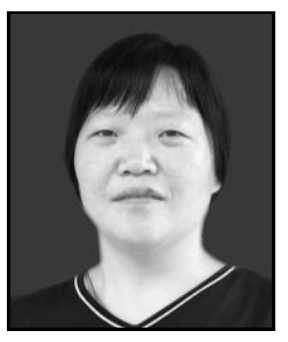

Zhang Feng-li, female, born on 6th, April , 1963, Han natínonality, Doctor, Professor, a tutor for doctors, works at Universily of Electronic Science and Technology School of Computer Science, main research directions: the database, the syncretisn of ambulation data. E-mail: fzhang@uestc.edu.cn

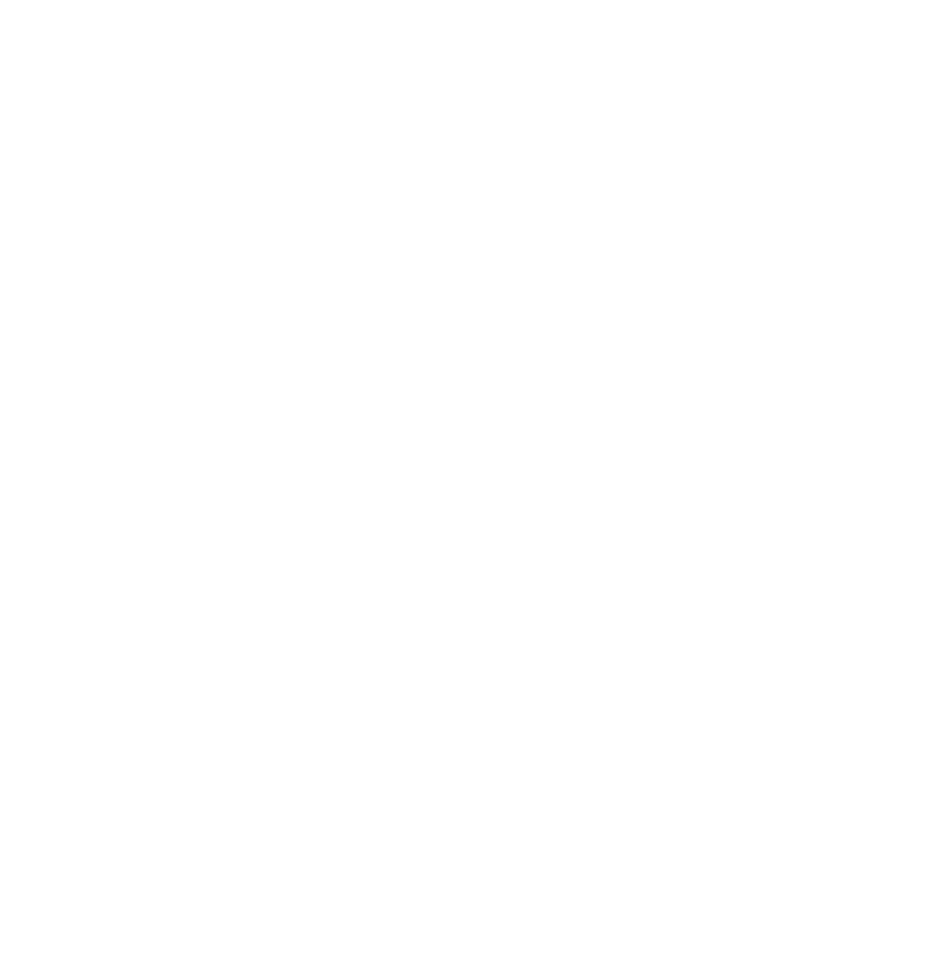


International Journal of Multimedia and Ubiquitous Engineering

Vol.9, No.8 (2014)

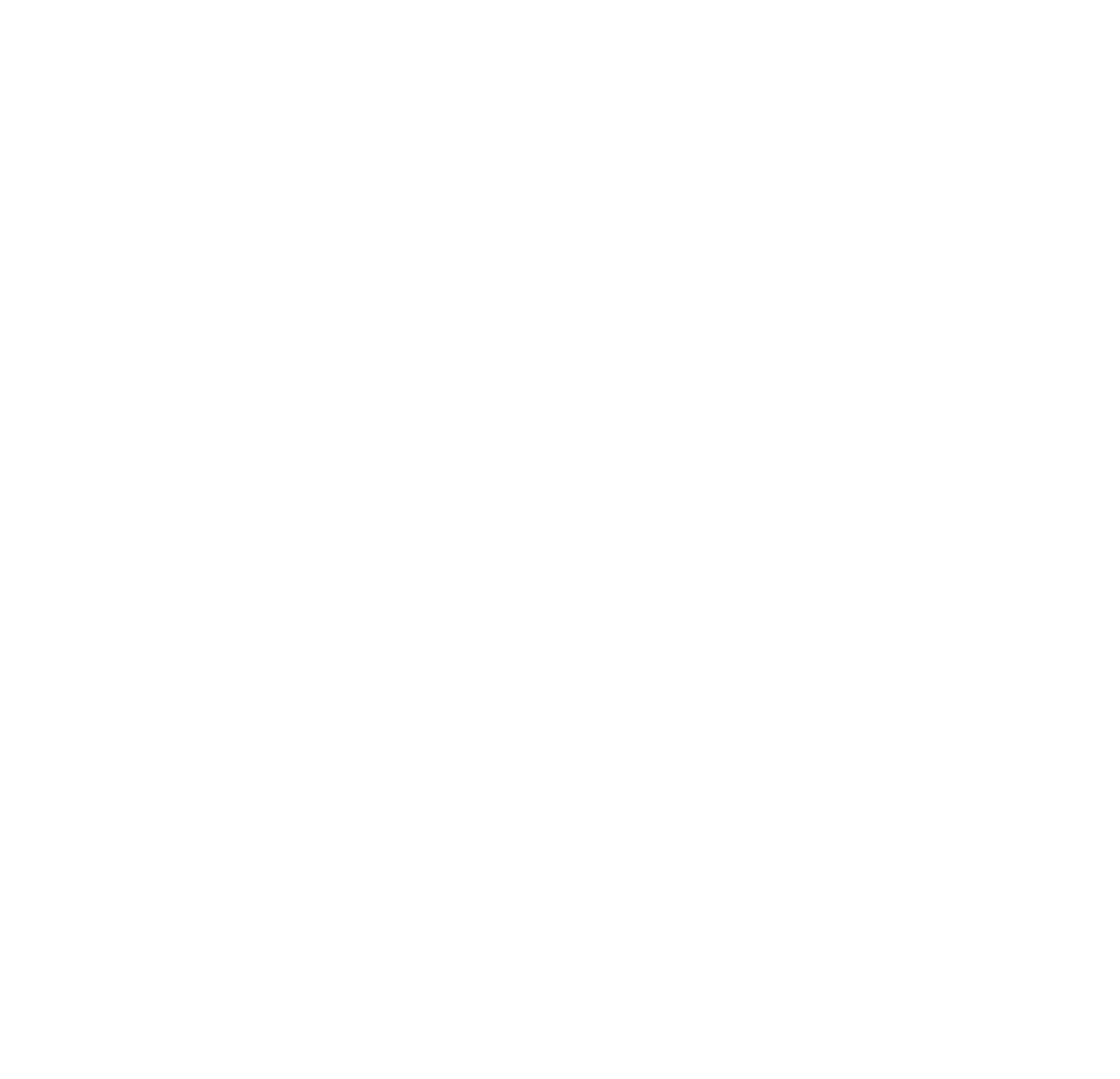

